\title{
Объективная оценка периоперационных осложнений у пациентов с отягощенной операционной травмой
}

Л. М. Смирнова, М. В. Смирнова

Национальный медицинский университет имени А. А. Богомольца, г. Киев

\section{Objective estimation of perioperative complications in patients, suffering complicated operative trauma}

\author{
L. M. Smyrnova, M. V. Smyrnova \\ Bogomolets National Medical University, Kyiv
}

\section{Реферат}

Цель. Разработать единые правила объективной оценки периоперационных осложнений путем количественного определения нарушений энергоструктурных взаимоотношений в массе клеток организма.

Материалы и методы. В исследовании приняли участие 35 пациентов с поздними осложнениями органов брюшной полости воспалительного характера. До операции состояние пациентов было оценено как ASA III функциональный класс (дисфункция). Во время операции был использован метод внутривенного наркоза целевыми концентрациями пропофола с искусственной вентиляцией легких в режиме нормовентиляции. Оперативное лечение заключалось в удалении очага воспаления, санации брюшной полости с последующим дренированием. Длительность оперативного вмешательства - $(153 \pm 21)$ мин. Объем кровопотери не превышал 10\% объема циркулирующей крови. Методом энергобиометрического мониторинга оценивали безопасность лечебных мероприятий, которая определяла результаты и исход медицинской помощи.

Результаты. В отличие от методов оценки обычно мониторируемых показателей вспомогательных систем, энергобиометрический мониторинг является чувствительным методом определения метаболических изменений в организме пациента в периоперационном периоде.

Выводы. Оценка энергоструктурного взаимоотношения в массе клеток организма служит интегральным маркером анализа текущего состояния и дальнейшего прогноза эффективности лечения. Исход лечения прямо пропорционально зависит от сроков устранения нарушений энергоструктурного дефицита.

ключевые слова: эффективность лечения; компьютерная программа; энергобиомониторинг; энергоструктурные взаимоотношения в массе клеток организма; энергокоррекция.

Abstract

Objective. To elaborate the universal rules of objective estimation of perioperative complications, using quantitative determination of disorders of the energy-structural interrelationship in the organism cellular mass.

Materials and methods. In the investigation 35 patients took part, who have late inflammatory complications in the abdominal cavity organs. Preoperatively the patients' state was estimated as ASA III functional class (dysfunction). During the operation the method of intravenous narcosis was applied, using the targeted concentrations of propofol with artificial pulmonary ventilation in regime of normoventilation. Operative treatment consisted of the inflammatory focus elimination, the abdominal cavity sanation with subsequent drainage. Duration of operative intervention was $(153 \pm 21) \mathrm{min}$. The blood loss volume did not exceeded $10 \%$ of the circulating blood volume. Security of the treatment measures, determining results and summation of the medical help was estimated, using the energy-biometrical monitoring.

Results. Unlike the estimation method of traditionally monitored indices of supporting systems, the energy-biometric monitoring constitutes a sensitive method of the metabolic changes determination in a patient organism in perioperative period. Conclusion. Estimation of the energy-structural interrelationship in the organism cellular mass serves the integral marker of analysis for current state and further prognosis of the treatment efficacy. The treatment result depends in direct proportion from the terms of elimination of the energy-structural deficiency disorders.

Keywords: efficacy of treatment; computeric program; energy-biomonitoring; the energy-structural interrelationship in the organism cellular mass; the energy correction.

В эпоху всеобщей роботизации и научных разработок в области создания искусственного интеллекта логично возникает повышенное желание анестезиологов прогнозировать результаты периоперационного анестезиологического обеспечения, которое неразрывно связано с разработкой и совершенствованием методов индивидуальной оценки процесса лечения [1]. К наиболее популярным направлениям применения искусственного интелекта относятся прогнозирование различных ситуаций, оценка циф- ровой информации с попыткой дать по ней заключение, а также анализ различных данных с поиском скрытых закономерностей. Компьютеры прочно вошли в нашу жизнь. Поэтому разработка и усовершенствование расчетно-диагностических программ для использования в работе врача-анестезиолога становятся реальностью нашего времени. Однако широкие возможности систем телекоммуникации, к сожалению, в минимальном объеме используются в медицинской практике. Необходимо подчеркнуть, что 
для компьютерной оценки динамики лечебного процесса и безопасности пациента необходима смена интеллекта врачей, только при этом условии инновационные технологии будут востребованы в полном объеме [1, 2].

В процессе жизни человек постоянно подвергается воздействию неблагоприятных факторов. Любое повреждающее воздействие приводит к возникновению компенсаторных реакций организма, направленность которых обеспечивает сохранение функциональной и морфологической структуры организма. Без ответа защитных механизмов на повреждение организм обречен на гибель. Согласно принципу «причинности» заболевания формируются вследствие неспособности организма либо противостоять внешним повреждающим механизмам, либо их компенсировать [2]. Следовательно, возникает необходимость оценки степени напряженности компенсаторных резервов по отношению к силе повреждающего фактора.

Объективность оценки тяжести состояния больного является необходимым инструментом для принятия решения о тактике периоперационного лечения и решения вопроса о его качестве. Создание единых правил периоперационной медицины позволит более качественно оценивать состояние энергоструктурных взаимоотношений в массе клеток организма (МКО) и ориентировать врача на выбор оптимального для конкретного пациента метода лечения с прогнозированными результатами в послеоперационном периоде [3, 4]. Базовые стандарты анестезиологического мониторинга, применяемые ко всем видам анестезии, объемные, не совсем точные и в экстремальных ситуациях могут приводить к ситуационным ошибкам. Поэтому текущая информация о пациенте должна быть дополнена четкими критериями оценки и мерами обеспечения безопасности [5, 6]. Приоритетными должны стать хорошо интерпретированные методы анализа массива текущих данных для объяснения гипотезы рекомендуемого к принятию решения [6]. Отсутствие навыков применения систем поддержания принятия врачебных решений ограничивает расширение стандартов по обеспечению безопасности пациента и не гарантирует конкретных прогнозированных результатов лечения. Базовые стандарты анестезиологического обеспечения подлежат пересмотру, что может быть оправдано развитием новых технологий и практики [6].

Мониторинг является одним из обязательных и ключевых компонентов анестезии. Тем не менее, во время анестезии некоторые методы стандартного мониторинга могут оказаться клинически не значимыми для обнаружения неблагоприятных инцидентов [7]. Основой для разработки и усовершенствования современных методов периоперационной медицины должен стать принцип достаточности функциональных резервов. При этом объективная оценка энергоструктурных взаимоотношений в МКО является необходимым инструментом для принятия решения о тактике лечения.

В рамках нового концептуального подхода к биологической целостности организма разработан метод коли- чественной оценки метаболических изменений в организме, предназначение которого - коррекция текущего энергетического дефицита для объективной оценки степени периоперационной выживаемости пациентов. Приоритетной составляющей энергобиометрического мониторинга (ЭБМ) является показатель потребности организма в энергетическом обеспечении. Способ определения потребности организма в кислородном обеспечении закреплен авторским правом [4, 8, 9]. Предложенный метод позволяет объективно оценивать функциональное состояние пациента, определять безопасность используемого комплекса периоперационной медицины, устанавливать опасность применяемых лечебных средств и действий, оценивать взаимосоответствующее энергетическое сопряжение МКО, которое способно удовлетворить основную биологическую потребность человека динамическое самообновление.

Научно обоснованная составляющая метода придает надежность доказательной медицине и служит научной базой для разработки собственно медицинской информатики с реализацией ее достижений в цифровой медицине [8 - 11].

Философией поставленной цели является представление о взаимосоответствующем энергетическом сопряжении МКО, удовлетворяющем основную биологическую потребность организма - динамическое самовосстановление как проявление жизнеспособности организма. Реализация метода ЭБМ, являющегося частью программы «Аудит витализма», предполагает вычисление должного, текущего и необходимого (потребность) уровня потребления кислорода в соответствии с разработанным алгоритмом при помощи математических выражений. Принцип метода основан на сравнении текущего энергопроизводства с уровнями его надежности и максимальных возможностей. Предназначение метода - количественное определение нарушений энергоструктурных взаимоОтношений в МКО и своевременное их устранение. Эффективность лечения повышается за счет одновременного лечения пациента и болезни. Количественные различия расчетных показателей отражают градиентное несоответствие величин энергетического обеспечения. Кроме точного определения уровня дефицита энергии и своевременной его коррекции, стало возможным объективно определять степень выживаемости пациентов и их способность к динамическому самовосстановлению. Значения доставки и потребления кислорода являются точными критериями прогноза выживаемости [5, 8-10].

Информационная система «Аудит витализма» содержит современные расчетные данные и разработана для врачей, которые сталкиваются с расчетами от случая к случаю для пациентов разных возрастных групп. Интерфейс простой и понятный, отображающий энергоструктурные взаимоотношения в МКО в режиме, приближенному к реальному времени. Диалог с системой осуществляется с клавиатуры компьютера. Результаты расчетов точно в цифровом виде выдаются по мере введения измененных переменных величин в короткий срок, что по- 
зволяет создавать план ведения анестезии или корректировать его, по мере необходимости, в кратчайшие сроки. Цифровые мониторы, составляющие основу «мониторинга безопасности», и компьютерные системы позволяют минимизировать так называемый человеческий фактор, являющийся более чем в $2 / 3$ наблюдений причиной анестезиологических ошибок и осложнений. Программа предназначена для персонального компьютерного оборудования, ее применение не требует специального обучения. Описан ответ клеток организма на стимуляцию и повреждение. Теоретические разработки, которые положены в основу программы “Аудит витализма”, изложены в монографиях Г. А. Шифрина 2016 и 2018 гг. [3 - 5]. Текстовые комментарии для правильного понимания и трактовки полученных результатов вычисления в рамках персонального мониторинга прилагаются к программе “Аудит витализма" в виде монографии «Персонификация периоперационной безопасности» [5].

Цель исследования: разработать единые правила объективной оценки периоперационных осложнений путем количественного определения нарушений энергоструктурных взаимоотношений в МКО.

\section{Материалы и методы исследования}

Работа выполнена по результатам периоперационного аудита энергоструктурных взаимодействий в МКО, резервов, свойств и эффективности ауторегуляции у пациентов с поздними осложнениями органов брюшной полости воспалительного характера. В проспективном и ретроспективном исследованиях приняли участие 35 пациентов. Состояние пациентов до операции оценили как ASA III функциональный класс. Энергоструктурные взаимодействия в МКО соответствовали дисфункции. В условиях внутривенного наркоза целевыми концентрациями пропофола с искусственной вентиляцией легких в режиме нормовентиляции выполняли удаление очага воспаления, санацию брюшной полости с последующим ее дренированием. Длительность оперативного вмеша-

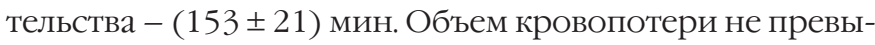
шал 10\% объема циркулирующей крови.

В периоперационном периоде проводили исследование глубокой картины кислородного режима и фактора компенсации потребности в транспорте кислорода, кислотно-основного и водно-электролитного балансов, показателей гемодинамики и микроциркуляции крови, непрямое определение основного обмена. Неинвазивный мониторинг осуществляли при помощи интегрированного монитора Primus Infinity Delfa (Dräger). Газовый состав крови определяли на аппарате "ABL 800” («Radiometr», Дания).

Статистическую обработку клинического материала проводили с помощью описательной статистики, пакета прикладных программ «Microsoft Excel 2007» и «STATISTICA® for Windows 6.0». Достоверность значений оценивали согласно $\mathrm{t}-$ критерию Стьюдента для n=35. Полученные результаты считали значимыми при уровне достоверности р меныше 0,05 для t больше 2,0796.

Безопасность лечебных мероприятий, которая определяла результаты и исход медицинской помощи, оценивали методом ЭБМ. Референтные и средние значения показателей ЭБМ представлены в табл. 1.

Клиническое применение информационной системы “Аудит витализма” основано на сравнении текущего энергопроизводства с уровнями его надежности и максимальных возможностей, то есть на количественном определении возможности организма отвечать на внешнюю агрессию любой этиологии. Количественные различия значений потребления кислорода зависят от индивидуальных реакций организма на внешние воздействия. В табл. 1 приведены референтные значения потребления кислорода в соответствии со стрессовыми реакциями организма и свойственным им нарушением кислородного режима.

Энергетическое обеспечение каждая клетка организма осуществляет строго индивидуально. Энергопродукция точно соответствует изменяющейся активности кле-

\begin{tabular}{|c|c|c|c|}
\hline Таблица 1. & МКО в соответ & иническим со & реакцией \\
\hline \multirow{3}{*}{ Клинический статус - (стресс-реакция) } & \multicolumn{3}{|c|}{ Уровни } \\
\hline & активности & готовности & потребности \\
\hline & 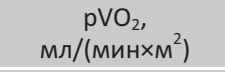 & 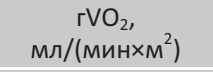 & $\begin{array}{c}\pi \mathrm{VO}_{2} \\
\left.\mathrm{мл/(мин} \times \mathrm{M}^{2}\right)\end{array}$ \\
\hline Стресс-активация & $\begin{array}{c}\text { от } 148 \text { до } 170 \\
(160,39 \pm 8,16)\end{array}$ & & $\begin{array}{c}\text { от } 152 \text { до } 178 \\
(165,74 \pm 6,58)\end{array}$ \\
\hline Дисфункция - (стресс-реализация) & $\begin{array}{c}\text { от } 112 \text { до } 147 \\
(128,61 \pm 7,24)\end{array}$ & & $\begin{array}{l}\text { от } 118 \text { до } 151 \\
(134,52 \pm 3,77)\end{array}$ \\
\hline Недостаточность - (стресс-повреждение) & $\begin{array}{c}\text { от } 86 \text { до } 111 \\
(92,41 \pm 5,68)\end{array}$ & $\begin{array}{c}110-140 \\
(126,47 \pm 4,33)\end{array}$ & $\begin{array}{c}\text { от } 91 \text { до } 117 \\
(103,37 \pm 4,62)\end{array}$ \\
\hline Несостоятельность - (стресс-разрушение) & $\begin{array}{c}\text { от } 54 \text { до } 85 \\
(72,32 \pm 6,17)\end{array}$ & & $\begin{array}{c}\text { от } 56 \text { до } 90 \\
(73,49 \pm 6,34)\end{array}$ \\
\hline Несостоятельность - (стресс-дезинтеграция) & $\begin{array}{c}\text { от } 34 \text { до } 53 \\
(43,58 \pm 5,12)\end{array}$ & & $\begin{array}{c}\text { от } 35 \text { до } 55 \\
(44,39 \pm 7,26)\end{array}$ \\
\hline \multicolumn{4}{|c|}{$\begin{array}{ll}\text { Примечание. } & \mathrm{pVO}_{2}-\text { реальное потребление кислорода тканями организма; } \mathrm{rVO}_{2}-\text { должный уровень потребления } \\
& \text { кислорода тканями организма; пVO } \mathrm{O}_{2}-\text { потребность организма в потреблении кислорода (для расчета } \\
& \text { привлекали показатель } \mathrm{C}_{x} \text {, который определяли на аппарате AVL } 800 \text { (фирмы «Radiometr», Дания). } \\
& \text { То же в табл. 2, 3. }\end{array}$} \\
\hline
\end{tabular}


ток, что строго поддерживается аппаратом генома, если страдает энергоструктурная синхронизация. Поэтому обмен веществ в МКО имеет разные уровни интенсивности.

Уровень активности (А) - интенсивность процессов обмена в функционирующих клетках организма, которые изменяются в соответствии с текущим уровнем метаболической активности $\left(\mathrm{pVO}_{2}\right.$ - реальное потребление кислорода тканями организма).

Уровень готовности (Г) - интенсивность метаболизма, которую должна поддерживать неактивная в данный момент МКО для сохранения способности немедленного и неограниченного функционирования $($ гVО - должный уровень потребления кислорода тканями организма). Такой уровень является необходимым, например, для процессов поддержания определенной разницы концентрации ионов натрия и калия в условиях стресса. Следовательно, таким уровнем считается уровень должного (ДОО) или индивидуального основного обмена, соответствующий возрасту (годы), росту (см), массе тела (кг). ДОО определяли в соответствии с общеизвестным математическим выражением (кДж /сутки).

Уровень потребности (П) - необходимая интенсивность метаболизма, которая является достаточной для сохранения морфоструктурного баланса в организме (пVO $\mathrm{V}_{2}$ - потребность организма в потреблении кислорода). Если эта потребность не удовлетворяется, в клетках организма наступают необратимые изменения и танатогенез. Пример: на микроанализаторе газов крови (RapidLab-248 фирмы «Bayer») измеряли базовые показатели кислотно-основного состояния, затем вносили дополнительные данные в компьютерную программу. Расчетные, количественные данные переменных (активность, готовность, потребность) высвечивались на мониторе в отдельном окне.

Следует отметить, что при любой интенсивности обмена ведущее значение имеют следующие факторы: процесс энергетического обеспечения клеток; физико-химическое состояние клеточной массы и структура мембран клеток, активность ферментов, баланс ионов и электрофизиологические параметры; изменения генетической программы клеток и (или) процессов ее реализации; нарушение механизмов энергоструктурного сопряжения.

Расстройство энергоструктурной синхронизации в МКО является «пусковым» моментом и одним из механизмов их альтерации как при ишемии, так и в период восстановления кровотока, что является неотъемлемой составляющей любого метода анестезиологического обеспечения. Причины возникающих нарушений могут быть разными, например, тканевой тип гипоксии обычно обусловлен нарушением способности тканей «поглощать» кислород из крови. При этом утилизация кислорода тканями может затрудняться из-за угнетения биологического окисления различными ингибиторами, нарушения синтеза ферментов или повреждения мембранных структур клетки. При гистотоксической гипоксии ткани не в состоянии извлекать кислород из тканевых капилля- ров даже при высоком его содержании в крови. Следовательно, независимо от причины возникают расстройства энергоструктурной синхронизации в МКО с последующей гибелью клеток. В свою очередь уменьшение МКО и нарушение функции оставшихся клеток способствуют увеличению количества осложнений.

\section{Результаты}

У 35 пациентов, которые были оперированы по поводу поздних осложнений органов брюшной полости воспалительного характера, до начала операции функциональное состояние было оценено как дисфункция энергоструктурного взаимодействия в МКО. Сознание ясное. Кожа сухая и бледная. Слизистые оболочки влажные. Частота дыхания - в среднем (18 \pm 5$)$ за 1 мин, среднее артериальное давление - $(12,39 \pm 0,27)$ кПа, частота сердечных сокращений - от 70 до 125 за 1 мин. Насыщение капиллярной крови кислородом колебалось в пределах от 94 до 97\%, уровень гемоглобина - от 111 до 130 г/л. Общее потребление кислорода МКО составило (127,94 \pm $2,97)$ мл/(мин $\left.\times \mathrm{M}^{2}\right)$, реальная доставка кислорода $\left(\mathrm{pDO}_{2}\right)$ в исходном состоянии - $(515,39 \pm 13,21)$ мл/ $\left(\right.$ мин $\left.\times \mathrm{m}^{2}\right)$, что в среднем на $(19,64 \pm 2,49) \%$ превышало возможности организма в реальном потреблении кислорода и было на $(14,59 \pm 0,69) \%$ меньше должной величины. Показатель потребности в доставке кислорода $\left(\mathrm{nDO}_{2}\right)$ в исходном состоянии составил в среднем $(594,42 \pm 25,18)$ мл/ $\left(\right.$ мин $\left.\times \mathrm{M}^{2}\right)$ и не отличался от должного уровня. Лабораторные данные также соответствовали состоянию дисфункции, которое характеризуется прежде всего нарушением стресс-устойчивости пациентов к внешней агрессии. Эти нарушения обусловлены нозоиндуцированным повреждением энергоструктурного гомеостаза. В связи с тем что у пациентов уровень потребности в потреблении кислорода преобладал над реальным его потреблением, состояние энергоструктурного взаимоотношения в МКО расценивали как нестабильное. Поэтому рекомендованный алгоритм для коррекции энергоструктурного взаимоотношения в МКО - статус-стабилизация. Метод направлен на устранение дефицита воды, электролитов, белка, гемоглобина с последующим удовлетворением суточной потребности в них. В результате лечения обычно удается достичь уровня реального потребления кислорода в МКО, который может соответствовать должному уровню потребления кислорода тканями организма, что отвечает потребности организма в кислородном ресурсообеспечении.

Изучена динамика показателей энергоструктурного взаимоотношения в МКО у пациентов в условиях тотальной внутривенной анестезии на основе целевых концентраций пропофола (табл. 2).

Как видно из данных в табл. 2, в соответствии с видами биологической целостности организма у пациентов до начала операции имела место стресс-активация. Это функциональное состояние организма, при котором уровень энергоструктурного взаимоОтношения в МКО можно 


\begin{tabular}{|c|c|c|c|c|}
\hline \multirow[t]{2}{*}{ Таблица 2.} & \multicolumn{4}{|c|}{$\begin{array}{l}\text { Динамика потребления кислорода МКО }(\bar{x} \pm \mathrm{m}) \text { у пациентов с поздними осложнениями органов } \\
\text { брюшной полости воспалительного характера }(\mathrm{n}=35) \text { в условиях тотальной внутривенной } \\
\text { анестезии на основе целевых концентраций пропофола (дисфункция) }\end{array}$} \\
\hline \multirow{3}{*}{\multicolumn{2}{|c|}{ Этапы наблюдения }} & \multicolumn{3}{|c|}{ Уровни } \\
\hline & & активности & готовности & потребности \\
\hline & & $\begin{array}{c}\mathrm{pVO}_{2} \\
\left.\mathrm{Mл/(Mин} \times \mathrm{M}^{2}\right)\end{array}$ & $\begin{array}{c}\mathrm{rVO}_{2} \\
\mathrm{M} /\left(\mathrm{MUH} \times \mathrm{M}^{2}\right)\end{array}$ & $\begin{array}{c}\Pi \mathrm{VO}_{2} \\
\mathrm{M} /\left(\mathrm{MиH} \times \mathrm{M}^{2}\right)\end{array}$ \\
\hline Должные вел & ичины & $178,46 \pm 2,51$ & & $177,33 \pm 9,12$ \\
\hline Дисфункция & исход) & $127,94 \pm 2,97$ & & $157 \pm 12$ \\
\hline Индукция & & $122,88 \pm 2,26$ & & $142 \pm 7$ \\
\hline Начало опер & ции & $112,00 \pm 2,39$ & & $182 \pm 6$ \\
\hline Основной эта & & $87,99 \pm 3,02$ & $124,19 \pm 2,53$ & $121 \pm 10$ \\
\hline Конец операь & & $93,08 \pm 3,28$ & & $135 \pm 8$ \\
\hline \multicolumn{5}{|c|}{ Сутки после операции } \\
\hline $1-e$ & & $139,94 \pm 2,71$ & & $138 \pm 9$ \\
\hline $2-e$ & & $105,92 \pm 2,88$ & & $146 \pm 7$ \\
\hline $3-n$ & & $142,67 \pm 3,12$ & & $165 \pm 5$ \\
\hline
\end{tabular}

описать математическим выражением: уровни активности и потребности превышают уровень готовности (А > Г $<$ П). В таких условиях функциональные возможности организма достаточны для самовосстановления, однако диапазон безопасности колеблется в пределах от 15 до 19\%, что требует высокой точности определения безопасности каждого компонента экстрацеллюлярной витализмпротекции. Тем более, что показатели энергоструктурного взаимоотношения в МКО соответствовали гиподинамической дисфункции, которая чревата гипоэргической опасностью и является следствием сформировавшейся биоэнергетической недостаточности (БЭН). Во время операции дополнительные нарушения энергетического обмена, вызванные как самой травмой, так и фармакологическими препаратами, способствуют формированию недостаточности или несостоятельности энергоструктурного взаимодействия в МКО. БЭН является результатом любого осложнения и различной степени его выраженности. Гипоэргоз манифестирует обширность выключения поврежденной части МКО, что достоверно отображает показатель энергоструктурной активности $\left(\mathrm{pVO}_{2}\right)$. Между тяжестью операционной травмы, осложнениями и БЭН существует прямо пропорциональная зависимость. В результате совместного влияния на организм пациентов операционной травмы и анестезии изменения энергоструктурной активности МКО достигали уровня недостаточности на основном этапе и в конце операции, невзирая на то, что среднее артериальное давление достоверно не отличалось от исходного значения и составляло соответственно $(11,86 \pm 0,67)$ и $(12,60 \pm 0,27)$ кПа. Снижение модальности анестезии и адекватная хирургическая санация способствовали восстановлению энергетического взаимоотношения в МКО до уровня, который превышал исходные значения. Тем не менее, в течение периода наблюдения восстановления энергоструктурной активности МКО не произошло как из-за депрессии системы транспорта кислорода, так и из-за нарушения энергоструктурных взаимоотношений в МКО.
Потребность в потреблении кислорода на всех этапах исследования, кроме основного этапа операции, была выше уровня готовности. При значениях пVО 2 выше 148 и меньше 192 мл/(мин × м²) формируется энергоструктурная безопасность (ЭСБ), индекс которой можно рассчитать при помощи математического выражения: [(пVО 2 148) : $\left.\Pi \mathrm{VO}_{2}\right] \times 100, \%$.

На тех этапах исследования, когда величина пVО 2 преобладала над величиной г $\mathrm{VO}_{2}$, энергоструктурные взаимоотношения в МКО были адаптированы к внешним воздействиям. Тем не менее, на основном этапе операции величина г $\mathrm{VO}_{2}$ преобладала над величиной п $\mathrm{VO}_{2}$ с последующим формированием деструктивных отношений в энергоструктурной сопряженности.

Потребность в доставке кислорода (пDО 2 ) на всех этапах клинического наблюдения была выше $\mathrm{pDO}_{2}$, что свидетельствует об ограничении функциональной активности системы транспорта кислорода под воздействием внешних факторов, таких как операционная травма и наркоз. Необходимо подчеркнуть, что на всех этапах исследования величины $\mathrm{pDO}_{2}$ и пDО 2 были ниже должных и исходных величин и не достигали исходного уровня в течение 3 сут после операции.

Характер реакций организма на внешние факторы и их качественно-количественная характеристика определяются результатом попарного сопоставления результатов вычисления показателей активности-готовностипотребности. Изменения энергоструктурных взаимоотношений в МКО у пациентов в условиях тотальной внутривенной анестезии на основе целевых концентраций пропофола представлены в табл.3.

Попарным сопоставлением результатов количественного вычисления показателей активности-готовностипотребности определяли характер реакций организма на внешние факторы, присваивая им качественно-количественную характеристику.

Стресс-реактивность организма определяется сравнением между собой показателей степени активности (А) и 


\begin{tabular}{|c|c|c|c|c|c|c|}
\hline \multirow{4}{*}{ Показатели, \% } & \multicolumn{6}{|c|}{$\begin{array}{l}\text { Изменения энергоструктурных взаимоотношений в МКО }(\bar{x} \pm m) \text { у пациентов с поздними } \\
\text { осложнениями органов брюшной полости воспалительного характера }(n=35) \text { в условиях } \\
\text { тотальной внутривенной анестезии на основе целевых концентраций пропофола }\end{array}$} \\
\hline & \multicolumn{6}{|c|}{ Этапы наблюдения } \\
\hline & \multirow{2}{*}{ до операции } & \multirow{2}{*}{$\begin{array}{c}\text { начало } \\
\text { операции }\end{array}$} & \multirow{2}{*}{$\begin{array}{c}\text { основной } \\
\text { этап операции }\end{array}$} & \multirow{2}{*}{$\begin{array}{c}\text { конец } \\
\text { операции }\end{array}$} & \multicolumn{2}{|c|}{ сутки после операции } \\
\hline & & & & & $1-\mathrm{e}$ & $2-\mathrm{e}$ \\
\hline $\mathrm{A} / \Gamma$ & $3,12 \pm 0,49$ & $-10,71 \pm 1,29 *$ & $-42,52 \pm 3,94 *$ & $-33,34 \pm 2,35^{*}$ & $11,42 \pm 1,07^{*}$ & $-18,07 \pm 1,72 *$ \\
\hline$\Pi / \Gamma$ & $21,07 \pm 0,85$ & $31,67 \pm 2,43^{*}$ & $-2,47 \pm 0,44^{*}$ & $8,14 \pm 0,72 *$ & $10,14 \pm 1,15^{*}$ & $15,05 \pm 1,36^{*}$ \\
\hline А/П & $-22,65 \pm 2,11$ & $-62,53 \pm 3,16^{*}$ & $-39,13 \pm 2,35 *$ & $-45,16 \pm 2,83^{*}$ & $1,42 \pm 0,21 *$ & $-39,04 \pm 2,48 *$ \\
\hline
\end{tabular}

готовности (Г) организма ответить на внешний раздражитель. Позитивный результат вычисления (А > Г), когда преобладает активность над готовностью, свидетельствует о достаточных возможностях организма отвечать на внешнее воздействие без повреждения. У пациентов до начала операции соотношение величин $\mathrm{pDO}_{2} / \mathrm{\Gamma DO}_{2}$ было позитивным и составило в среднем $(3,12 \pm 0,49) \%$. Если результат негативный $(\mathrm{A}<\Gamma)$, реакции организма на внешнее воздействие определяются как ареактивные, а возможности адаптационных систем организма исчерпанные или ограниченные. Именно такую ситуацию наблюдали на этапах операции (см. табл.3) - изменения показателя были существенными и достоверными. Адекватная хирургическая коррекция и снижение модальности анестезии в 1-е сутки после операции способствовали повышению потребления кислорода МКО, а значит, и адаптационных возможностей.

Соотношение показателя активности потребления кислорода $\left(\mathrm{pVO}_{2}\right)$ и потребности в потреблении кислорода (пVО $\mathrm{V}_{2}$ количественно указывает направление развития адаптационных реакций организма. Если $\mathrm{pVO}_{2}$ превышает п $\mathrm{VO}_{2}$, а результат вычисления математического выражения (А - П) : А позитивный (+), \%, преобладают процессы стабильности. Если результат вычисления математического выражения (А - П) : А негативный (-), \%, энергоструктурное взаимоотношение в МКО становится нестабильным. У пациентов с поздними осложнениями органов брюшной полости воспалительного характера в условиях тотальной внутривенной анестезии на основе целевых концентраций пропофола на всех этапах наблюдения отмечали преобладание потребности в потреблении кислорода над реальной величиной потребления кислорода $(\mathrm{A}<$ П). Следовательно, невзирая на стабильность показателей центральной гемодинамики, формировалась нестабильность в энергоструктурном взаимодействии МКО.

Преобладание пDО 2 над г $\mathrm{DO}_{2}$ на всех этапах периоперационного наблюдения характеризует адаптивность организма к внешнему и нозоиндуцированному повреждающему фактору. Снижение повреждающего воздействия в кратчайшие сроки будет способствовать восстановлению энергоструктурных взаимоотношений в МКО и выздоровлению пациента с минимальными повреждениями всех систем организма. Любое действие врача должно быть направлено на достижение необходимой для пациента энергоструктурной активности. Поэтому методоло- гия определения безопасности периоперационной медицины, нарушений ауторегуляции, значимости резервов и свойств энергоструктурной активности становится необходимой в ежедневной работе врача.

\section{Обсуждение}

В результате анализа клинических исследований пациентов с поздними осложнениями органов брюшной полости воспалительного характера стало понятным, что предложенная информационная система оценки энергоструктурных взаимоотношений в МКО позволяет определять пути ликвидации нарушений энергоструктурного гомеостаза организма, что является оптимальным условием для достижения самовосстановления в периоперационном периоде. В периодической литературе нет сведений о применении информационных программ для оценки степени нарушения биологической устойчивости организма к внешним воздействиям. Предложенный метод в цифровом обозначении подтверждает тот факт, что периоперационные изменения в организме есть ни что иное, как проявление патологических состояний энергоструктурного взаимодействия в МКО. Тщательная оценка возможности компенсаторных резервов, обеспечивающих выживаемость организма в периоперационном периоде, позволяет анестезиологу обоснованно выбрать оптимальные методы анестезии и интенсивной терапии курируемому пациенту. Предложенный метод наглядный, информативный и эффективный, поэтому считаем его применение обоснованным и целесообразным.

Предложенная нами информационная программа, как и существующие, адаптирована к формату Excel. Функционально работа с программой визуализируется на одном экране персонального компьютера. Однако для эффективной работы программы необходимо в специально выделенные окна вносить дополнительные данные требуемых клинико-биохимических параметров организма, которые включают: общепринятые клинико-биохимические анализы, насыщение кислородом артериальной крови. Также необходимы сведения о массе тела, росте, величине артериального давления, частоте дыхания и пульса, температуре тела обследуемого. «Ввод данных» пациента осуществляется на определенную дату и время. Полученные данные оценочной системы автоматически переносятся в выделенное окно-таблицу расчета показателей активности, готовности, потребности организма в энергетиче- 
ском обеспечении. Введение новых данных отражает изменения энергоструктурных взаимоотношений в МКО в соответствии с алгоритмами, заложенными в логику работы программы. Все данные, необходимые для работы программного обеспечения, используются в режиме, приближенном к реальному времени. В отличие от существующих программ, компьютерный анализ данных пациента позволяет оценить индивидуальные энергоструктурные взаимоОтношения в МКО, адаптационные возможности отдельных органов и систем, возможности самовосстановления, нарушенного как основным заболеванием, так и операционной травмой. Определение категории энергоструктурной активности позволяет применять соответствующую энергопротективную технологию интенсивной терапии и придать периоперационному обеспечению персонифицированный характер. Применение энергопротективных технологий интенсивной терапии существенно улучшает результаты лечения пациентов.

Преимущество программы заключается в выявлении на ранних этапах групп риска, определении оптимального и әффективного варианта профилактики и лечения.

Особенности компьютерно-аналитической системы: использование предложенного программного обеспечения, как и других медицинских программ, предполагает наличие персонального компьютера, функционирующего под управлением операционной системы Microsoft Windows XP, и адаптированность аналитической системы к компьютерной программе Excel; универсальность и простота применения компьютерных программ не требуют дополнительного обучения персонала введению исходных параметров, необходимых для последующих расчетов; индивидуальный подход к пациенту не является новым, однако отсутствие влияния человеческого фактора обеспечивает максимальную объективность и точность расчета показателей, характеризующих адаптационные возможности организма пациентов, позволяет проводить динамическое наблюдение за эффективностью лечения и прогнозировать исход, что стимулирует поддержку принятия врачебного решения; для эффективной работы с аналитической системой необходима надлежащая специальная подготовка врача-специалиста для правильной интерпретации данных оценочной системы, основанной на биологической целостности организма, а правильно интерпретированные данные объясняют соответствующие гипотезы предложенного программой решения; обладание научными основами виталологии и работа с информационной системой “Аудит витализма” обеспечат ускоренное выздоровление пациентов и позволят увеличить продолжительность здоровой жизни до ее биологического предела.

\section{Выводы}

1. ЭБМ является чувствительным методом определения метаболических изменений в организме в отличие от методов оценки обычно мониторируемых показателей вспомогательных систем.
2. Оценка энергоструктурного взаимоотношения в МКО служит интегральным маркером анализа текущего состояния и дальнейшего прогноза эффективности лечения.

3. Исход и эффективность лечения прямо пропорционально зависят от времени устранения нарушений энергоструктурного дефицита.

\section{Подтверждение}

Финансирование. За счет авторов работы.

Информация об участии в работе. Смирнова Л. М. - дизайн исследования, сбор и обработка клинического материала, анализ полученных данных и написание текста; Смирнова М. В. - обработка клинического материала.

Конфликт интересов. Авторы, принявшие участие в исследовании, прочитали и одобрили окончательный вариант рукописи и декларируют отсутствие конфликта интересов относительно данной рукописи.

Согласие на публикацию. Оба автора дали согласие на публикацию данной рукописи.

\section{References}

1. Gusev AV, Dobridnyuk SL. Iskusstvennyy intellekt v meditsine i zdravookhranenii Informatsionnoye obshchestvo. 2017;4-5:78-93. [In Russian]

2. Belousov AN. Novaya kompiuternaya programma otsenki tiazhesti patsiyenta. In: Materialy II Ukrainsko-Rosiiskoho konhresu «Aktualni pytannia anesteziolohii ta intensyvnoi terapii 24-26 travnia., m. Odesa. Bil, zneboliuvannia i intensyvna terapiia. 2012;(1d):28-30. [In Ukrainian].

3. Shifrin GA, Tumanskiy VA, Kolesnik YuM. Vitalologiya. Zaporozhye: Dikoye Pol; 2018. 288 p. [In Ukrainian].

4. Smyrnova LM, Shyfrin HA, Serikov KV. vynakhidnyky; Kyiv. Shalimov National Institute of Surgery and Transplantology, patentovlasnyk. Sposib vyznachennya potreby orhanizmu v enerhetychnomu zabezpechenni. Patent Ukainy No 71931. 2012 Lip 25. [In Ukrainian].

5. Shifrin GA. Personifikatsiia perioperatsionnoy bezopasnosti. Zaporozhye: Dike Pole; 2016. 88 p. [In Russian].

6. Neznanov A. Khorosho interpretiruyemyye metody analiza dannykh, fev. 2018. Available from: https://www.youtube.com/watch?v=5k7KCzEFL4I. [In Russian].

7. Yovenko IA, Kobelyatskiy YU, Tsarev AV, Kuzmova YeA, Mashin AM. Gemodinamicheskiy monitoring v praktike intensivnoy terapii kriticheskikh sostoyaniy. Meditsina neotlozhnykh sostoyaniy. 2016;5(76):42-6. doi: 10.22141/2224-0586.5.76.2016.76433. [In Russian].

8. Smyrnova LM. Bioenergeticheskaya nedostatochnost' anesteziologicheskogo obespecheniya. Bil, zneboliuvannia i intensyvna terapiia. 2016;4:5965. doi: 10.25284/2519-2078.4(77).2016.94332. [In Ukrainian].

9. Smyrnova LM. Opasnost povrezhdeniya adaptatsionnykh mekhanizmov pri anesteziologicheskom obespechenii operatsiy po povodu zabolevaniy organov pankreatoduodenalnoy zony. Bil, zneboliuvannia i intensyvna terapiia. 2017;(4):73-8. [In Ukrainian]. doi: 10.25284/25192078.4(81).2017.119320.

10. Gotovtsev AV. Opredeleniye BPK i koeffitsiyenta skorosti biokhimicheskogo potrebleniya kisloroda: monitoring, pryamaya i obratnaya zadachi, formuly, raschety i tablitsy. Vodnyye resursy. 2016;43(6):633-47. [In Russian].doi: 10.7868/S0321059616050060.

11. Posternak GI, Tkacheva M, Litovka RV, Solenyy BS. Raschetno-diagnosticheskiye programmy v anesteziologii i intensivnoy terapii. Meditsina neotlozhnykh sostoyaniy. 2011;(6):101-3. [In Ukrainian].

Надійшла 25.05.19 